\title{
Aa. Vv., Romans à clés. Les ambivalences du réel, sous la direction de Anthony Glinoer et Michel Lacroix
}

\section{Marta Baravalle}

\section{(2) OpenEdition}

1 Journals

Édition électronique

URL : http://journals.openedition.org/studifrancesi/678

DOI : 10.4000/studifrancesi.678

ISSN : 2427-5856

Éditeur

Rosenberg \& Sellier

Édition imprimée

Date de publication : 1 avril 2015

Pagination : 213-214

ISSN : 0039-2944

\section{Référence électronique}

Marta Baravalle, «Aa. Vv., Romans à clés. Les ambivalences du réel, sous la direction de Anthony Glinoer et Michel Lacroix », Studi Francesi [En ligne], 175 (LIX | I) | 2015, mis en ligne le 01 avril 2015, consulté le 18 septembre 2020. URL : http://journals.openedition.org/studifrancesi/678; DOI : https://doi.org/ 10.4000/studifrancesi.678

Ce document a été généré automatiquement le 18 septembre 2020

\section{(c) (†) $\odot$}

Studi Francesi è distribuita con Licenza Creative Commons Attribuzione - Non commerciale - Non opere derivate 4.0 Internazionale. 


\title{
Aa. Vv., Romans à clés. Les ambivalences du réel, sous la direction de Anthony Glinoer et Michel Lacroix
}

\author{
Marta Baravalle
}

\section{RÉFÉRENCE}

Aa. Vv., Romans à clés. Les ambivalences du réel, sous la direction de ANTHONY GLINOER et MICHEL LACROIX, Liège, Presses Universitaires de Liège, 2014, Collection «Situations», vol. 2, pp. 203.

1 Le recueil naît d'une journée d'études du GREMLIN (Groupe de recherche sur les médiations littéraires et les institutions) sur la nécessité de renouveler la question du rapport entre fiction et réalité dans le cadre littéraire contemporain des «vies d'écrivain». Les propositions qui suivent la Présentation des éditeurs représentent des tentatives de décodage de différents procédés mis en œuvre dans la difficilement atteignable définition du roman à clés. Le double jeu d'encryptage et décryptage propre au genre pris en examen serait donc significatif d'un travail spécifique sur les discours littéraires et remettrait en cause les enjeux de la référentialité auteur-personnage.

Michel LACROIX entame son essai Imaginaire, légendaire, fictif: romans à clés et fiction de la vie littéraire (pp. 11-20) en donnant quelques exemples d'écrivains transposés dans un roman à clés, expression d'une curiosité croissante autrement décrite à la fois comme effet et cause du voyeurisme du lecteur et de la culture de la célébrité. Lacroix propose d'opérer une distinction entre les différentes images d'auteur, construites dans et par le discours (ou, pour mieux dire, dans sa réception), dont le référent réel n'offrirait qu'un modèle d'inspiration. La fictionnalisation jouerait donc un rôle fondamental dans l'évolution possible des figures légendaires en figures imaginaires. L'écrivain 
légendaire serait une figure telle que Rimbaud, acteur solitaire de la vie littéraire, auquel s'opposeraient les écrivains imaginaires tels que les poètes maudits, interprètes d'une posture collective.

Dans Réalité, fiction et plaisir. Introduction du livre "The Art of Scandal. Modernism, Libel law and the Roman à Clef" (pp. 21-42), Sean LATHAM s'adonne à la tâche de démonter l'autonomie de la fiction du biographique par le biais d'une relecture de la littérature moderne à travers le prisme du roman à clés. En établissant une taxonomie bien que provisoire du genre, l'auteur souhaite ainsi émanciper le roman à clés de toutes questions morales qui le hantent depuis sa naissance et jusqu'au $\mathrm{xx}^{\mathrm{e}}$ siècle. À ses origines, la littérature à potins marquait l'entrée d'un certain plaisir pour l'écriture et la lecture, dont Sean LATHAM défend la légitimité. L'A. construit enfin un parallèle entre les scandales surgis autour de Dorian Gray et d'Ulysse et le débordement dans les domaines juridiques et éthiques causé de nos jours par la réception du roman à clés, ce qui mettrait en évidence le besoin de renouveler le débat sur l'interdépendance du fictif et du réel.

4 La réflexion sur le réalisme conservateur réside au cœur de l'enquête menée par Mathilde BOMBART aussi. Romans à clés: une pratique illégitime au filtre de la critique littéraire des journaux (pp. 43-65) est effectivement le compte rendu d'un travail de recherche duré dix ans principalement à partir de la base Europresse.com dans la presse hebdomadaire et quotidienne française parue en France. L'étude a poussé l'A. à explorer d'avantage la nature du rapport contemporain à la littérature, notamment du point de vue de la rentabilité envisagée par son exploitation journalistique, et à formuler une hypothèse sur le rôle déterminant du lecteur.

5 La métaphore du cénacle sert efficacement à Anthony GLINOER et Vincent LAINSNEY (Les illusions perdues, ou les romans cénaculaires, pp. 67-85) afin de montrer les ambivalences fictionelles et fonctionnelles du roman à clés. Les romans cénaculaires du XIX ${ }^{e}$ siècle auraient effectivement un publique préétabli, prêt à accueillir les suggestions mystiques offertes par le récit d'un groupe oligarchique, intellectuellement qualifié et moralement autotélique, qu'on voudrait connaître de l'intérieur. L'écrivain cénaculaire $\mathrm{y}$ dramatiserait le phénomène social à faveur d'un enrichissement narratologique des personnages et il contribuerait ainsi à démarquer l'image répandue collectivement du vrai cénacle.

6 Dans Figurations et médiogrammes. Les microfictions du "Petit Bottin des Lettres et des Arts" (pp. 87-97), Denis SAINT-AMAND introduit deux instruments d'observation du roman à clés. Le concept de médiogramme expliquerait le mécanisme désacralisant propre au Petit Bottin, œuvre rédigée à huit mains par Fénéon, Méténier, Moréas et Adam, tandis que la figuration, «construction sémiotique d'un sujet individualisé» dans un contexte spécifique, contribuerait à la formule d'une naturalisation manquée. L'hybridité de l'intertexte livresque et de l'intertexte auctorial devient ainsi une priorité dans l'étude du rapport entre l'auteur et ses personnages.

7 Plaisir d'offrir, joie de recevoir. Le roman à clé décadent de Rachilde (pp. 99-112) de Micheal R. FINN offre un compendium des œuvres dédiées à et écrites par Marguerite Eymery. À la fois auteur et cible, d'un côté Rachilde aurait dirigé la construction de son personnage social par le biais de son écriture, en s'authentifiant et en s'auto-identifiant dans un groupe dont les référents étaient autant transparents que possible. De l'autre côté, elle 
a été l'objet à peine caché de nombreuses œuvres satiriques, de ce fait méritant l'appellation de reine des romans à clés.

8 Elisheva ROSEN, spécialiste de la Recherche, s'adonne à une étude visant à qualifier les subtiles correspondances entre l'œuvre de Proust et le genre du roman à clés. La thèse de La pratique des clés au prisme de la "Recherche" (pp. 113-130) met en relief l'incontestable richesse du roman en exposant une volonté forte de la part de l'auteur, qui jouerait à provoquer les lecteurs en anticipant leurs réactions pour la simplicité du plaisir de la lecture. Le lecteur serait donc partie intégrante du jeu, dans un projet littéraire qui brouille savamment les cartes entre l'art et la vie.

Paradoxalement, dans "Les Mandarins", les clés pour se dire (pp. 131-144) Anne STRASSER rappelle le mépris de Simone de Beauvoir pour le genre du roman à clés, dont pourtant son roman avait été qualifié, pour s'interroger sur les frontières de la mise en scène de la vie littéraire. Une réflexion sur toute l'œuvre autobiographique de Simone de Beauvoir s'impose dès lors que l'A. avance dans l'analyse des croisements entre le temps de l'écriture et le temps de la vie de l'écrivain pour proposer la consécration d'une place inéluctablement dédiée à la création des lecteurs.

10 À partir d'une remarque sur la représentativité inhérent à tout texte, Le théâtre sans son double: jouir sans le dire dans "Scènes d'enfants" de Normand Chaurette (pp. 145-157) de Richard SAINT-GELAIS propose quelques intéressantes considérations sur les rapports entre spécularité et référentialité. L'étude repose sur la distinction des cadres qui régissent et l'interprétation de la fiction et l'interprétation des faits en s'appuyant sur le récit d'une représentation théâtrale tout-à-fait particulière. Encore une fois, le lecteur est trompé par un auteur qui s'amuse à pervertir le dispositif théâtral en exposant les mécanismes qui le règlent.

11 En s'éloignant momentanément du domaine strictement littéraire, Jean-Pierre ESQUENAZI offre son point de vue sur l'un des long métrages les plus célèbres de Woody Allen dans Lecture des opérations de référentialité. L'exemple de "Manhattan" (pp. 159-168). L'analyse du film se joue autour des opérations référentielles qui affectent la réception par le biais de l'introduction d'un double de l'auteur et finalement propose une taxonomie des spectateurs basée sur les réactions de ceux-ci aux entrecroisements d'histoire et discours.

12 Selon Alexandre GEFEN, l'œuvre de Puech serait à considérer comme un roman de la vie de la théorie littéraire. Il argumente sa théorie dans Benjamin Jordane, roman: jeux identitaires et aventures métatexuelles dans l'œuvre de Jean-Benoît Puech (pp. 169-178), article qui remet en cause le dispositif de fictionnalisation de Puech, déployé par successions d'hétéronymes interposés. Indiqué sous le nom de biofiction, l'ensemble des œuvres rendrait effectivement manifeste une dialectique entre textualismes formalistes et finalités de la littérature en passant à travers des questions d'ordre ontologique.

Dans Organisations secrètes. La Gauche prolétarienne dans la littérature française contemporaine (pp. 179-193), Mathilde BARRABAND se penche sur les œuvres qui recueillent de vrais ou de faux récits de vies vécues dans les environnements de la Gauche prolétarienne dans les années 1960-1970. La question de la référentialité est vite portée en surface à partir du moment où l'on se demande si c'est l'auteur ou le lecteur à déterminer le genre des romans à clés et quelles pourraient être les fonctions sociologiques d'un système de codage et décodage élaboré en commun. 